\title{
Visual texture segregation based on orientation and hue
}

\author{
TARA C. CALLAGHAN, MARIA I. LASAGA, and W. R. GARNER \\ Yale University, New Haven, Connecticut
}

\begin{abstract}
We investigated visual texture segregation, using a task for which reaction time to locate a discrepant quadrant in an array of 36 elements was the dependent measure. Two dimensions of segregation were used: line orientation (horizontal vs. vertical, horizontal vs. left diagonal, and left vs. right diagonal) and hue ( 9 vs. 7 Munsell color steps). Levels on these two dimensions were varied singly to produce control arrays, in orthogonal combination to measure possible interference effects, and in redundant combination to measure possible facilitation effects. Segregation for control arrays was much more difficult when the two diagonal lines produced regions of texture than when arrays used lines involving one or two main axes, and 7-step hue-difference arrays were more difficult to segregate than 9 -step arrays, as expected. Orientation and hue were equally effective in producing fast texture segregation when used with optimal levels. The pattern of interference and facilitation for orthogonal and redundant combinations depended on the particular pair of line orientations used to produce texture: With horizontal and vertical lines, there was symmetric interference of each dimension on the other, as well as a redundancy gain. With horizontal and left diagonal lines, there was asymmetric interference of hue variation on orientation segregation, and no redundancy gain. With the two diagonals, there were symmetric interference and strong redundancy gains. These results clarify why different line orientations provide different effectiveness in texture segregation, and suggest that both interstimulus confusability (as with the two diagonals) and intrastimulus stability (as with horizontal and vertical lines) properties determine the ability of a dimension to interfere with, or to be interfered with by, another dimension in texture segregation.
\end{abstract}

Visual texture segregation is a perceptual process that involves the grouping together of elements on the basis of similarity, and the detection of boundaries between different regions of texture in the visual field. Beck (1982) defined texture as a visual pattern that is composed of similar elements within a common region of the visual field, so that the whole pattern appears to have a characteristic lightness, coarseness, or so forth. Texture segregation is easily produced by differences between regions in simple properties such as orientation (Beck, 1966, 1967, 1972; Olson \& Attneave, 1970), hue (Callaghan, 1984; Treisman, 1982; Treisman \& Gelade, 1980), or form (Treisman, 1982; Treisman \& Gelade, 1980). Complex properties, such as the particular arrangement of horizontal and vertical line segments (e.g., T vs. L; Beck, 1966, 1967,1972 ) or a specific conjunction of hue and form elements (e.g., red and square; Treisman \& Gelade, 1980 ), produce either difficult or no texture segregation.

This research was conducted at Yale University, where the first author was supported by a Natural Sciences and Engineering Research Council of Canada postdoctoral fellowship. Additional support came from National Institute of Mental Health Grant MH 14229 to W. R. Garner. Requests for reprints should be sent to Tara C. Callaghan, who is now with the Department of Psychology, Saint Francis Xavier University, Antigonish, N.S., Canada B2G 1C0; W. R. Garner, Department of Psychology, Box 11A, Yale Station, New Haven, CT 06520; or Maria I. Lasaga, who is now with the Department of Psychology, Gilmer Hall, University of Virginia, Charlottesville, VA 22901
Beck $(1972,1982)$ offered a model that can account for the discrepancy in ease of segregation between simple and complex properties. This model proposes that textural segmentation involves a preliminary unitization of the array elements. That is, features of elements are linked into "hyperfeatures" that serve as the actual elements for texture perception. For example, in an array that segregates into regions of Ls and Ts, vertical and horizontal lines are linked to form Ls and Ts; this linkage prevents the vertical and horizontal lines from serving as textural elements. These textural elements (in this case, Ls and Ts) are then aggregated into textural regions. Thus, if complex properties are used to produce texture segregation, and if complex properties involve a linking process, then segregation should be more difficult than it is when simple properties that do not require a linking process are used.

Treisman (1982; Treisman \& Gelade, 1980) offered an alternative explanation that is based on the distinction between preattentive and attentive processing. According to her model of figural perception, differences in the effectiveness of stimulus properties in producing segregation depend on whether any conjunction of features is necessary. During preattentive processing, parallel registration of features across the visual field occurs. For detailed analysis of an object of perception, attention and serial processing are required to integrate attributes of stimuli that are registered independently. 
In line with the work of Beck $(1972,1982)$ and Treisman (1982; Treisman \& Gelade, 1980), it is generally agreed that the apprehension of fields or regions in a multielement array is a process that occurs prior to detailed analysis of element information. Kahneman (1973) referred to this as the "unit formation" stage of figure perception; Neisser (1967) used the term "preattentive processing' to describe this stage. A basic premise associated with this view is that preattentive processing is an early, automatic stage of perception (Beck, 1972, 1982; Kahneman, 1973; Neisser, 1967; Treisman, 1982; Treisman \& Gelade, 1980). It follows that preattentive processing should not be easily influenced by other factors in the visual field. However, in recent research it has become increasingly evident that texture segregation based on simple property differences between regions is influenced by extraneous factors. In the present experiment, we explored the nature of this interference, using line orientation and hue differences to produce textured arrays.

\section{Segregation of Single Lines Based on Orientation}

The simple property of line orientation has been found to produce robust segregation when orientation differences exist across regions of single-line elements. The ease of segregation depends on the particular orientation difference that is present in the array. Olson and Attneave (1970) found that textures made up of vertical and horizontal lines produce better segregation than textures made up of different diagonal lines, even though the physical difference between the orientation of line elements in both cases is identical. Beck (1972) reported that the detection of a horizontal line in a four-element display that contained three vertical lines was better than detection of a left diagonal (i.e., a line rotated $45^{\circ}$ counterclockwise from vertical) in a background of three right diagonals (i.e., lines rotated $45^{\circ}$ clockwise from vertical). He concluded, therefore, that superior texture segregation for horizontal and vertical lines may be the result of the preattentive visual system's greater sensitivity to the difference between horizontal and vertical lines than to that between diagonal lines.

\section{Segregation of a Conjunction of Line Orientation and Hue}

When observers are required to segregate visual regions on the basis of a conjunction of line orientation and color, performance is impaired relative to performance of segregation on the basis of single-line differences. Beck (1982) reported that when observers were asked to count, for example, the number of blue diagonals in a background of blue vertical and red diagonal lines, mean counting time was significantly longer than in the control condition, in which blue diagonals were embedded in blue verticals. This result was not due to the simple background nonuniformity of color. In order to segregate blue diagonals in the first condition, observers had to abstract one particular conjunction of properties (i.e., blue and diagonal) from the others, a requirement which signifi- cantly interfered with segregation. Treisman and Gelade (1980) found similar results for hue and form (shape of letter) properties. When complex conjunctions of properties are used to produce texture, performance may be impaired because the basis for segregation (i.e., simple property differences between regions of texture) has been eliminated. However, in order to truly explore the nature of interference in segregation, it is necessary to study the influence of extraneous factors under conditions in which segregation based on simple property differences is possible.

\section{Segregation Based on Simple Property Differences with Random Variation of Second Property}

Callaghan (1984) devised an alternative procedure for investigating interference in texture segregation based on the stimulus manipulations of Garner and Felfoldy (1970). In Callaghan's study, a single property difference, either hue or brightness, defined the regions of texture. Either the second property was held at a constant level across elements in the entire array, or levels of the second property were alternated irrelevantly across the array. Callaghan found symmetric interference of one property on the other when component dimensions were of equivalent discriminability. That is, irrelevant variation of hue interfered with segregation based on brightness, and irrelevant variation of brightness interfered with segregation based on hue.

The finding of symmetric interference with hue and brightness dimensions is consistent with results from attention experiments that employ speeded classification tasks (Garner, 1974; Garner \& Felfoldy, 1970; Shepp, 1983). In the attention experiments, hue and brightness have been called integral dimensions (Garner \& Felfoldy, 1970) because separate processing of component dimensions is not possible. Callaghan's (1984) finding of symmetric interference using texture segregation, which is generally agreed to be a preattentive task, raises interesting implications. Specifically, it may be that hue and brightness dimensions are processed as integral dimensions even at the preattentive level of texture segregation. Alternatively, the finding of symmetric interference at both levels of processing may imply that a distinction between preattentive and attentive processing is not a useful one, at least insofar as texture segregation is concerned. The implications of Callaghan's results would be clarified by an investigation of interference effects using dimensions that can be processed separately in attention tasks (i.e., separable dimensions).

\section{Purpose of the Present Experiment}

The present experiment was intended to further investigate the nature of interference and of dimensional interaction in texture segregation. The theories of Beck (1972, 1982) and Treisman (1982; Treisman \& Gelade, 1980) suggest that if properties do not need to be conjoined to produce texture elements in segregation, they should not interfere with each other when segregation is 
based on only one of the properties. The paradigm used in the present study was based on that used by Callaghan (1984). We used simple line segments that varied in orientation and in hue, because these dimensions appear to be separable in related tasks. Beck (1982, p. 299) reported a supplementary experiment which indicated that mean counting times for diagonal lines were equivalent whether the lines were embedded in a background of lines of uniform or nonuniform color. This finding suggests that line orientation and hue may be processed separably in the type of task used here. (See also Gottwald \& Garner, 1972, for evidence that hue and form are separable dimensions.)

In the present experiment, texture was defined by singleproperty differences or double-property differences. In single-property arrays, the level of the second property could be held constant (control arrays), or two levels of the second property could be varied randomly throughout the array (orthogonal arrays) to determine whether interference occurred. In arrays with double-property differences the two dimensions were used in a perfectly correlated way, so that segregation of regions of texture could be based on either dimension (redundant arrays), to determine whether facilitation occurred.

\section{METHOD}

\section{Observers}

Sixteen Yale undergraduates either were paid or received course credit for participation in this experiment. All participants had normal or corrected vision. Each observer took part in one experimental session lasting approximately $1.5 \mathrm{~h}$.

\section{Procedure}

On each trial, observers were presented with a 36-element stimulus array. Each stimulus array had a quadrant containing 9 elements that differed from the 27 background elements in orientation, in hue, or in a redundant combination of these two properties. Hypothetical arrays showing horizontal versus vertical lineorientation differences are presented in Figure 1. Reaction time (RT) to locate the discrepant quadrant was the dependent measure.

Observers initiated a trial by pressing a button switch held in the left hand after the experimenter gave a verbal ready signal. Upon initiation of a trial, the stimulus array was presented for $1,000 \mathrm{msec}$. The observer responded as quickly as possible by pressing one of four computer response buttons. The positions of the buttons corresponded to the positions of the four quadrants in a square. Between trials, the observer's index finger rested on a central button that was equidistant from each of the four response buttons.

A black fixation dot corresponding to the center of the stimulus array was in view when the stimulus was absent to provide general orientation. A trial lasted approximately $5 \mathrm{sec}$. On each trial, a computer measured RT, to the nearest millisecond, from the onset of the stimulus until the depression of the response button, and recorded RT along with errors and the stimulus presented.

\section{Stimulus Construction and Presentation}

The stimuli were $6 \times 6$ matrices (i.e., 36-element arrays) composed of line elements that varied in orientation and/or hue. Lines were drawn with colored markers on plastic vellum and mounted on white cardboard. Line thickness was $1 \mathrm{~mm}$ and line length was $8 \mathrm{~mm}$.

Stimuli were presented in a three-channel Scientific Prototype tachistoscope (Model GB). The stimuli were viewed at $127 \mathrm{~cm}$. The individual lines subtended a visual angle of $0.36^{\circ}$ for length, and

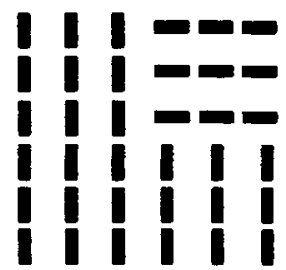

(a)

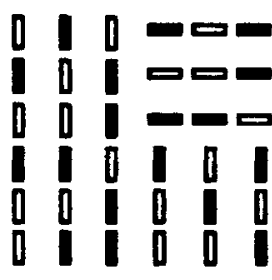

(b)

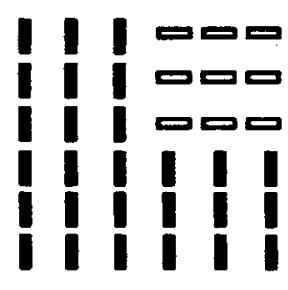

(c)
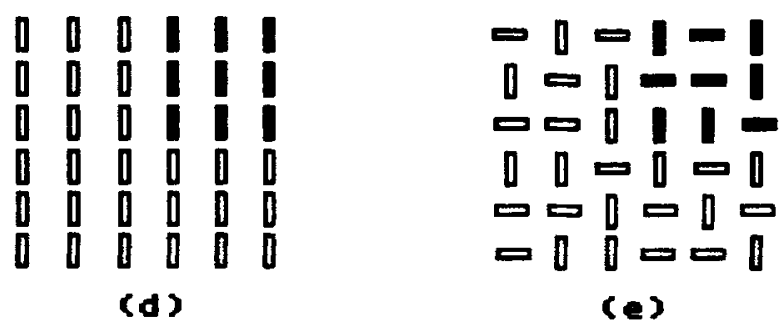

Figure 1. Hypothetical textured arrays with quadrant elements that differ from background elements in line orientation (horizontal vs. vertical), showing the difference between (a) control, (b) orthogonal, and (c) redundant arrays, and in hue (difference represented by filled and open bars), showing the difference between (d) control and (e) orthogonal arrays.

the entire square matrix never subtended a visual angle that was more than $3.06^{\circ}$ on a side. The background luminance was $34.2 \mathrm{~cd} / \mathrm{m}^{2}$.

Four line orientations were used: horizontal $(\mathrm{H})$, vertical $(\mathrm{V})$, left diagonal ( $\mathrm{L}, 45^{\circ}$ counterclockwise from vertical), and right diagonal ( $R, 45^{\circ}$ clockwise from vertical). Three hues were used: H1 (Panatone marker No. 485, approximately 10R 5/10 Munsell notation), H2 (Panatone marker No. 259, approximately 7.5P 5/10 Munsell notation), and $\mathrm{H} 3$ (Design marker violet-red-violet, approximately 2.5RP 5/10 Munsell notation).

\section{Stimulus Conditions}

The stimulus arrays for this experiment were of two major types.

Single-property arrays (control and orthogonal). Each singleproperty array allowed segregation according to either line orientation or hue.

The orientation arrays differed in the particular line-orientation difference that produced texture. There were three possible orientation differences: horizontal versus vertical (HV), horizontal versus left diagonal (HL), and left diagonal versus right diagonal (LR). For each of these pairs of orientations, each orientation was used as the discrepant element in half of the arrays.

Hue arrays differed in the particular hue difference that produced texture. Half of the hue arrays contained elements that differed in 
hue by 9 steps in the Munsell color system (H1 vs. H2), and half contained elements that differed by 7 steps in the Munsell system (H1 vs. H3). For each of these two possible hue differences, half of the arrays had discrepant quadrant elements of each hue level.

Texture segregation could occur only according to a single property in these types of arrays; however, the other property in the array could be either held constant (control arrays) or varied randomly (orthogonal arrays).

In orientation-control arrays (Figure 1a), the level of hue was held constant across all elements at one of its three possible levels. There were three possible orientation-control arrays: HV-control, HL-control, and LR-control. For each of these possibilities, arrays were constructed at each of the three hue levels: $\mathrm{H} 1, \mathrm{H} 2$, and $\mathrm{H} 3$.

For hue-control arrays (Figure 1d), the property of line orientation was held constant at one of its four possible levels across all elements in the arrays. There were two possible subtypes of hue control arrays: 9-step-control and 7-step-control. For each of these possibilities, four arrays were constructed using one of each of the four line orientations for elements in a given array: $\mathrm{H}, \mathrm{V}, \mathrm{L}, \mathrm{R}$.

For orientation-orthogonal arrays (Figure $1 b$ ), differences in line orientation produced texture, but two levels of hue varied in a random manner. In half of these arrays, the levels of hue that varied were $\mathrm{H} 1$ and $\mathrm{H} 2$. In the other half, the levels of hue that varied were $\mathrm{H} 1$ and $\mathrm{H} 3$. Both of these possibilities occurred for each of the three orientation differences, HV, HL, and LR, resulting in six possible orientation-orthogonal arrays: HV-orthogonal/9-step, HV orthogonal/7-step, HL-orthogonal/9-step, HL-orthogonal/7-step, LR-orthogonal/9-step, and LR-orthogonal/7-step.

In hue-orthogonal arrays (Figure 1e), differences in hue produced texture, but levels of line orientation varied randomly across the array. In one third of these arrays, the particular levels of orientation varied were $H$ versus $V$; in one third, $H$ versus $L$; and in the remaining third, $L$ versus $R$. These three possible orthogonal combinations occurred for each of the two hue differences, 9-step and 7-step, resulting in six possible hue-orthogonal arrays: 9-steporthogonal/HV, 9-step-orthogonal/HL, 9-step-orthogonal/LR, 7step-orthogonal/HV, 7-step-orthogonal/HL, and 7-step orthogonal/LR.

Two-property (redundant) arrays. The second type of stimulus array was one in which texture segregation could be based on orientation or hue differences or both (Figure 1c). This redundant combination of line-orientation (i.e., HV, HL, or LR) and huediscriminability (i.e., 9-step or 7-step) differences resulted in six redundant arrays: HV/9-step, HV/7-step, HL/9-step, HL/7-step, LR/9-step, and LR/7-step.

\section{Experimental Design}

One hundred twenty different stimulus arrays were used in this experiment. Forty-eight of these arrays could be segregated on the basis of line orientation ( 24 control and 24 orthogonal), 48 on the basis of hue difference ( 24 control and 24 orthogonal), and 24 on the basis of a redundant combination of orientation and hue.

Recall that the orientation-control arrays contained one of three levels of hue and the orientation-orthogonal arrays contained two levels of hue with either a 9- or a 7-step difference in the Munsell system. For purposes of obtaining a balanced design of control and orthogonal arrays, we produced half of the orientation-control arrays with hue levels corresponding to those used in orientationorthogonal/9-step arrays (i.e., $\mathbf{H} 1$ and $\mathbf{H} 2$ ), and half with hue levels corresponding to those used in the orientation-orthogonal/7-step arrays (i.e., $\mathrm{H} 1$ and $\mathrm{H} 3$ ). The number of orientation-control arrays having hue level $\mathrm{H} 1$ was therefore twice the number of those having hue level $\mathrm{H} 2$ or $\mathrm{H} 3$.

Each hue-control array was constructed with one of four line orientations $(\mathrm{H}, \mathrm{V}, \mathrm{L}$, or $\mathrm{R})$. In order to balance hue-control and hueorthogonal arrays, we produced 8 of the 24 hue-control arrays with line orientations corresponding to those in the hue-orthogonal/HV arrays (i.e., horizontal and vertical), 8 corresponding to those in hue-orthogonal/HL arrays (i.e., horizontal and left diagonal), and
8 corresponding to those in hue-orthogonal/LR arrays (i.e., left and right diagonal). This balancing resulted in twice as many hue-control arrays with horizontal and left diagonal lines as there were with vertical and right diagonal lines.

Each observer was presented with five blocks of trials, each of which began with 15 practice trials. In each block, all 120 possible stimuli were presented once, and the order of presentation was randomized. Each observer received a different random order of presentation, and this order was different across blocks for all observers.

\section{RESULTS}

The error rate was low (overall mean proportion of errors $=0.04$ ), and the pattern of errors was similar to that of the RT data. Therefore, only RTs for correct responses were considered for detailed analysis. The raw RT data for each observer's correct responses were combined across blocks according to array subtype, and median RTs were then calculated for these arrays.

A preliminary analysis determined that the specific level of the second property held constant for the control arrays had no effect on $\mathrm{RT}$. Therefore, data for all control conditions were combined for further analyses.

\section{Single-Property Orientation Arrays}

Control effects. A one-factor analysis of variance of orientation-control data (see Table 1 for mean RTs corresponding to this analysis) revealed a significant effect of orientation difference $[F(2,30)=35.46, p<.01]$. The Lindquist (1953) critical difference method was employed for a posteriori comparisons warranted by this analysis and all others. Post hoc analysis showed that mean RTs were equivalent for $\mathrm{HV}$ and $\mathrm{HL}$ control arrays, and that these were faster than mean RTs for LR control arrays.

Interference effects. To analyze the interference produced by random variation of hue levels on orientation segregation, we employed a subjects $x$ orientation difference $X$ subtype of stimulus array analysis of variance. (See Table 1 for corresponding mean RTs.) There was a main effect of subtype of stimulus array $[F(2,30)$ $=13.19, \mathrm{p}<.011$. Post hoc analysis indicated that average RTs were equivalent for orientation-orthogonal/9-step and orientation-orthogonal/7-step arrays, and that these were significantly longer than average RTs for orientationcontrol arrays.

In addition, the extent of interference due to hue variation depended on the particular orientation difference that defined segregation, as indicated by a significant orien-

Table 1

Mean Reaction Times (in msec) for Correct Segregation Responses in the Interference Analysis of Orientation Arrays

\begin{tabular}{lcccc}
\hline Orientation & \multicolumn{3}{c}{ Subtype of Stimulus Array } & \\
\cline { 2 - 4 } Difference & Control & $\begin{array}{c}\text { Orthogonal } \\
\text { (9-step) }\end{array}$ & $\begin{array}{c}\text { Orthogonal } \\
\text { (7-step) }\end{array}$ & Average \\
\hline HV & 551 & 567 & 565 & 561 \\
HL & 560 & 577 & 579 & 572 \\
LR & 690 & 819 & 787 & 765 \\
Average & 600 & 654 & 643 & 632 \\
\hline
\end{tabular}


tation difference $x$ subtype of stimulus array interaction $[F(4,60)=7.27, p<.01]$. A posteriori tests indicated that interference occurred $(p<.05)$ for all types of orientation difference, but that it was strongest $(p<.01)$ for LR arrays. Furthermore, for the LR arrays, interference was stronger for orientation-orthogonal/9-step arrays $(\mathrm{p}<.01)$ than for orientation-orthogonal/7-step arrays $(\mathrm{p}<.05)$.

As expected, there was a significant orientation difference main effect $[F(2,30)=38.05, p<.01]$. A posteriori tests revealed that RTs were equivalent overall for HV and $\mathrm{HL}$ arrays, and were faster for these arrays than for LR arrays, not only on the average, but for each array subtype.

\section{Single-Property Hue Arrays}

Control effects. A one-factor analysis of variance using hue-control RT data produced a significant effect of hue difference $[F(1,15)=35.53, \mathrm{p}<.01]$. (See Table 2 for relevant RTs.) This effect indicates that for hue-control arrays, segregation was significantly faster when hue levels were 9 Munsell steps apart than when they were 7 steps apart, as expected.

Interference effects. To analyze the interference of hue segregation produced by variation of line orientation, we employed a subjects $\times$ hue difference $\times$ subtype of stimulus array analysis of variance. (See Table 2 for corresponding mean RTs.)

The main effect of subtype of stimulus array was significant $[F(3,45)=4.97, p<.01]$. Post hoc analyses revealed that the variation of horizontal and vertical lines in hue-orthogonal/HV arrays and of left and right diagonals in hue-orthogonal/LR arrays produced significant interference of hue segregation on the average and at both levels of hue discriminability. In contrast, the variation of horizontal and left diagonal lines did not produce interference at either level of hue discriminability.

As expected, the interference analysis of variance revealed a main effect of hue difference $[F(1,15)=45.63$, $\mathrm{p}<.01]$. Post hoc tests showed that overall, segregation was significantly faster when hue levels were 9 steps apart than when they were 7 steps apart.

\section{Two-Property (Redundant) Arrays}

In order to assess redundancy gain effects, each observer's median RT for each type of redundant array was compared with the faster of his/her median control RTs in $t$ tests for matched groups. This procedure was used

Table 2

Mean Reaction Times (in msec) for Correct Segregation Responses in the Interference of Hue Arrays

\begin{tabular}{lccccc}
\hline & \multicolumn{5}{c}{ Subtype of Stimulus Array } \\
\cline { 2 - 6 } $\begin{array}{l}\text { Hue } \\
\text { Difference }\end{array}$ & Control & $\begin{array}{c}\text { Orthogonal } \\
\text { (HV) }\end{array}$ & $\begin{array}{c}\text { Orthogonal } \\
\text { (HL) }\end{array}$ & $\begin{array}{c}\text { Orthogonal } \\
\text { (LR) }\end{array}$ & Average \\
\hline 9-step & 566 & 575 & 562 & 599 & 578 \\
7-step & 642 & 653 & 642 & 657 & 649 \\
Average & 604 & 614 & 602 & 628 & 613 \\
\hline
\end{tabular}

Table 3

Mean Reaction Times (in msec) for Correct Segregation Responses in the Analysis of Redundancy Effects

\begin{tabular}{llcc}
\hline & & \multicolumn{2}{c}{ Array Subtype } \\
\cline { 3 - 4 } Orientation & Hue & & Fastest \\
Difference & Difference & Redundant & Control \\
\hline HV & 9 -step & 526 & 546 \\
& 7 -step & 537 & 549 \\
HL & 9 -step & 548 & 555 \\
& 7 -step & 555 & 560 \\
LR & 9 -step & 542 & 566 \\
& 7 -step & 572 & 632 \\
\hline
\end{tabular}

because observers differed in RT to the two dimensions, and a redundancy gain may be inferred only if RT to the redundant dimensions is faster than RT to the faster of the dimensions used singly. (See Table 3 for mean RTs corresponding to these analyses.)

The results of the $t$ tests indicated that a redundancy gain in performance was found when HV orientation differences were correlated with 9-step hue differences $(p<.01$ ), but not when they were correlated with 7-step hue differences. No redundancy gains were observed when HL orientation differences were correlated with either 9- or 7-step hue differences. Finally, strong redundancy gains were observed when LR orientation differences were correlated with both 9- and 7-step hue differences (both ps $<.01$ ).

\section{Summary}

In the present experiment, we found the ease of visual texture segregation to be dependent on the particular orientation differences that produced texture ( $\mathrm{HV}$ and $\mathrm{HL}$ were both better than LR) and on the degree of hue difference (a 9-step difference was better than a 7-step difference).

Additionally, when texture elements differed in $\mathbf{H}$ versus $\mathrm{V}$ line orientation, hue variation interfered with segregation. When texture elements differed in hue (regardless of the degree of difference), the variation of $\mathrm{H}$ and $\mathrm{V}$ elements significantly impaired performance. Furthermore, the redundant combination of HV differences with 9-step hue differences produced a significant gain in performance.

When texture elements differed in $\mathrm{H}$ versus $\mathrm{L}$ line orientation, hue variation also produced interference of segregation. However, the variation of $\mathrm{H}$ and $\mathrm{L}$ lines did not interfere with segregation based on hue, at either level of hue discriminability. In addition, no redundancy gain in performance was observed when HL differences were correlated with hue differences.

When textured regions were segregated on the basis of $L$ versus $R$ line orientation, performance was poor, relative to performance on HV and HL arrays. There was strong interference when hue was varied, at both 9 and 7 steps. In addition, the irrelevant variation of diagonals significantly impaired hue segregation. Robust redundancy gains occurred when LR differences were correlated with either hue difference. 


\section{DISCUSSION}

The goal of this experiment was to clarify the factors that operate in producing interference of texture segregation, a task generally considered to be accomplished with little effort at a preattentive level of processing (Beck, 1972, 1982; Kahneman, 1973; Neisser, 1967; Treisman, 1982; Treisman \& Gelade, 1980).

We found overwhelming evidence for interference of orientation segregation (regardless of particular orientation difference) when hue levels were varied randomly (regardless of hue level difference). There was also interference of hue segregation, but only when HV and LR lines were varied randomly, not when HL lines were varied. The postulation of a stimulus similarity mechanism can account for these interference patterns. The individual lines in the $\mathrm{HV}$ and LR pairs have a physical difference of $90^{\circ}$, whereas those in the HL pair have a difference of $45^{\circ}$. Interference was directly related to the physical similarity of the elements that were varied. This is also supported by the finding that when hue levels varied by 9 Munsell steps, interference of orientation segregation was more pronounced than when hue levels varied by 7 Munsell steps. Thus, our data provide strong evidence for the claim that an important determinant of interference in texture segregation is the physical dissimilarity of the elements that vary orthogonally to the texture elements.

Furthermore, the extent to which random variation of two levels across the visual field will produce interference is not predicted by how well those elements serve to produce texture. In our experiment, $\mathrm{HV}$ and $\mathrm{HL}$ orientation differences were equally effective in producing texture segregation in control arrays. In contrast, LR orientation differences were poor texture elements. Lasaga and Garner (1983) have shown that the two diagonal lines have unstable properties when presented alone, and are highly confusable when presented in pairs. It would seem that the unstable properties noted in individual diagonal lines transfer to the case in which lines are used to produce distinct regions in larger arrays. Thus, we have evidence to support the view that the factors operating in the production of textured regions are separate from those that interfere with the segregation of those regions.

In his model of textural segmentation, Beck (1982) argued that two components enhance the perception of texture: high dissimilarity of elements between different regions, and high similarity of elements within regions. The random variation of orientation or hue across orthogonal arrays decreases element similarity within regions, relative to control arrays. Thus, our finding that irrelevant variation of a second property produced a longer mean RT, on the average, for orthogonal than for control arrays confirms Beck's contention that interstimulus similarity within regions is important. However, the lack of strong redundancy gains with these stimuli, as well as with hue and brightness stimuli (Callaghan, 1984), calls into question the relative importance of between-region dissimilarity. In the present experiment, we found redun- dancy gains both when component dimensions were of equivalent discriminability (HV/9-step, LR/7-step) and when they were not (LR/9-step). Redundancy gains were not evident in all cases of equivalent discriminability of component dimensions (HL/9-step). At least for these stimuli and tasks, there does not seem to be a consistent rule that governs the operation of redundancy gain effects. In the present analysis, the grouping together of elements to form a region of texture (i.e., within-group similarity) is a stronger component in the segregation process than is the separation of one textured region from another.

Finally, the present results converge with recent findings (Callaghan, 1984) to suggest that segregation of textured arrays is a process that is easily interfered with by the variation of other properties in the stimulus array. This ease of interference raises questions about the nature of preattentive processing itself. If such processing, or texture segregation as a specific form of such processing, is an automatic process, one not requiring conscious attention, then how can it be so easily interfered with? As Treisman (1982; Treisman \& Gelade, 1980) has shown, texture segregation based on a required conjunction of features or dimensions takes longer than segregation based on single features, and it is easy enough to argue that the required conjunction changes texture segregation from an automatic, preattentive process to an attentive process. In the present experiment, however, segregation could always occur on the basis of a single dimension, and thus automatic, rapid processing was presumably possible.

\section{Alternative Interpretations}

There are a number of possible interpretations of the interference effect in texture segregation. First, since interference did occur in the present case, it is possible that the distinction between preattentive and attentive processing is really not that of a clear dichotomy. Rather, a faster process may be available when texture can be segregated on the basis of a single dimension, but this process itself may be slowed when irrelevant variation of a second dimension occurs. It seems likely from our results that there is a continuum extending from fast (possibly automatic) processing to the much slower processing that is required when dimensions must be conjoined to satisfy the task requirements. But if there is truly such a continuum, then "preattentive" does not mean "prior to attentive," but simply "faster than attentive." In this case, the usefulness of preattentive processing as a construct to account for texture segregation is certainly weakened.

Second, it may be that the distinction between preattentive and attentive processing is a real one, but that interference occurs in the early processing of separate dimensional information. This interpretation violates our traditional assumptions regarding the nature of preattentive processing (see Neisser, 1967; Treisman \& Gelade, 1980 ), and calls into question its utility as a theoretical construct.

Finally, it is possible that in this particular task, hue and line orientation may not be separable dimensions in the same sense that they are in attentional tasks (Gottwald 
\& Garner, 1972). In a typical attention task, speeded sorting, decks of single stimuli are sorted into piles according to a value on a single dimension (Garner \& Felfoldy, 1970). All possible values of stimulus dimensions are contained within orthogonal decks, but not within a single stimulus contained in that deck. This was not the case with the stimuli used in the present experiment. In our orthogonal arrays, all dimensional values were simultaneously present for each stimulus array. As Eriksen, O'Hara, and Eriksen (1982) argued, performance may be expected to differ depending on whether stimuli, and hence dimensional values, are presented singly or simultaneously. These researchers accounted for the finding of simultaneous same-different judgments by a continuousflow model of visual information processing that invokes the operation of a response-competition mechanism. Very briefly, the model proposes that when simultaneous samedifferent judgments are made, there is the possibility that both "same" and "different" responses are primed for each presentation. The degree of priming for a given response depends on such factors as feature overlap between targets and between targets and noise stimuli. Thus, when the physical similarity between two identical targets is judged, the "different" response will be primed insofar as the noise stimuli share few features with the targets. Likewise, when targets are different, the "same" response will be primed insofar as the targets themselves, and the targets and noise stimuli, share features. When applied to the present situation, in which textured arrays allow for four possible overt responses (corresponding to the four quadrants), the response-competition model makes the following predictions. In control arrays (e.g., Figure la), the gradual accumulation of information should lead to an unambiguous priming of the "upper right" response. In contrast, in orthogonal arrays (e.g., Figure 1b), information may gradually accumulate not only for the correct "upper right" response, but also for the three incorrect responses, due to the presence of irrelevant hue differences found in all quadrants. The priming of incorrect overt responses may therefore slow the decision process for orthogonal, as compared to control, arrays. This interpretation holds that the interference effects observed in this preattentive task may be due to the operation of a response-competition mechanism.
It will be the task of future research to assess whether the present results are due to one, or to some combination, of these alternative interpretations.

\section{REFERENCES}

BECK, J. (1966). Effect of orientation and of shape similarity on perceptual grouping. Perception \& Psychophysics, 1, 300-302.

Beck, J. (1967). Perceptual grouping produced by line figures. Perception \& Psychophysics, 2, 491-495.

BECK, J. (1972). Similarity grouping and peripheral discriminability under uncertainty. American Journal of Psychology, 85, 1-19.

BECK, J. (1982). Textural segmentation. In J. Beck (Ed.), Organization and representation in perception. Hillsdale, NJ: Erlbaum.

Callaghan, T. C. (1984). Dimensional interaction of hue and brightness in preattentive field segregation. Perception \& Psychophysics, 36, 25-34.

Eriksen, C. W., O'Hara, W. P., \& Eriksen, B. (1982). Response competition effects in same-different judgments. Perception \& Psychophysics, 32, 261-270.

GARNER, W. R. (1974). The processing of information and structure. Potomac, MD: Erlbaum.

GaRNER, W. R., \& FELFOLdY, G. L. (1970). Integrality of stimulus dimensions in various types of information processing. Cognitive Psychology, 1, 225-241.

GotTWALD, R. L., \& GARNER, W. R. (1972). Effects of focusing strategy on speeded classification with grouping, filtering, and condensation tasks. Perception \& Psychophysics, 11, 179-182.

Kahneman, D. (1973). Attention and effort. Englewood Cliffs, NJ: Prentice-Hall.

LASAGA, M. I., \& Garner, W. R. (1983). The effect of line orientation on various information processing tasks. Journal of Experimental Psychology: Human Perception \& Performance, 9, 215-225.

LINDQuist, E. F. (1953). Design and analysis of experiments in psychology and education. New York: Houghton Mifflin.

Neisser, U. (1967). Cognitive psychology. New York: AppeltonCentury-Crofts.

OLSON, R. K., \& ATTNEAVE, F. (1970). What variables produce similarity grouping? American Journal of Psychology, 83, 1-21.

SHEPP, B. E. (1983). The analyzability of multidimensional stimuli: Some constraints on perceived structure and attention. In T. J. Tighe \& B. E. Shepp (Eds.), Perception, cognition, and development: Interactional analyses. Hillsdale, NJ: Erlbaum.

Treisman, A. M. (1982). Perceptual grouping and attention in visual search for features and for objects. Journal of Experimental Psychology: Human Perception \& Performance, 8, 194-214.

Treisman, A. M., \& Gelade, G. (1980). A feature-integration theory of attention. Cognitive Psychology, 12, 97-136.

(Manuscript received January 25, 1985; revision accepted for publication November 26,1985 .) 